\title{
Portable cardiac self-stress test device development based on metabolic equivalent (MET)
}

\author{
Whomaira binti Abdul Samad, Mohd Afzan bin Othman, Norlaili binti Mat Safri, \\ Mohd Azhar bin Abdul Razak \\ School of Electrical Engineering, Faculty of Engineering, Universiti Teknologi Malaysia, Malaysia
}

\begin{tabular}{|c|c|}
\hline Article Info & ABSTRACT \\
\hline Article history: & \multirow{11}{*}{$\begin{array}{l}\text { Cardiovascular disease (CVD) is the main cause of death worldwide and the } \\
\text { type varies depending on symptom and causes. There are several procedures } \\
\text { to determine the type of CVD such as blood test, holter monitoring, } \\
\text { cardiac stress test and many more. Cardiac stress test is a procedure to exert } \\
\text { pressure on the heart by doing physical activities to determine heart } \\
\text { functionality and abnormal heart activity. It is done to gather information on } \\
\text { heart condition supported by evidence in the form of electrocardiogram } \\
\text { (ECG) signal. However, patients with heart problem will have to take turns } \\
\text { to run the test due to limited access to the equipment especially in } \\
\text { government hospital. Therefore, this paper proposed the development of } \\
\text { cardiac self-stress test device to provide a pre-diagnosis result based on } \\
\text { metabolic equivalent (MET) value. The device named "i-STress device" is a } \\
\text { stand-alone device integrating the use of software application and hardware. } \\
\text { The software was built by using MIT App Inventor } 2 \text { and the hardware built } \\
\text { based on Arduino } 101 \text { and Sparkfun Heart Monitor AD } 8232 \text { and the test } \\
\text { result saved in SD card. The device will be useful to sort out patient's case } \\
\text { priority as well as providing pre-diagnosis result before conducting a real } \\
\text { cardiac stress test. }\end{array}$} \\
\hline Received Dec 25, 2018 & \\
\hline Revised Mar 27, 2019 & \\
\hline Accepted Apr 18, 2019 & \\
\hline Keywords: & \\
\hline Cardiac self-stress test & \\
\hline Cardiovascular disease & \\
\hline ECG & \\
\hline MET & \\
\hline Metabolic equivalent & \\
\hline Stress test & \\
\hline
\end{tabular}
Copyright $@ 2019$ Institute of Advanced Engineering and Science.
All rights reserved. Copyright $@ 2019$ Institute of Advanced Engineering and Science.
All rights reserved.

\begin{abstract} type varies depending on symptom and causes. There are several procedures pressure on the heart by doing physical activities to determine heart heart condition supported by evidence in the form of electrocardiogram metabolic equivalent (MET) value. The device named " $\mathrm{i}$-STress device" is a The software was built by using priority as well as providing pre-diagnosis result before conducting a real
\end{abstract}

\section{Corresponding Author:}

Mohd Afzan bin Othman,

School of Electrical Engineering,

Faculty of Engineering,

Universiti Teknologi Malaysia,

81310 Skudai, Johor, Malaysia.

Email: afzan@utm.my

1. INTRODUCTION

Cardiovascular Disease (CVD) is a very common yet lethal disease. The American Heart Association (AHA) defined CVD as a disease caused by atherosclerosis which refers to a process where atheroma or commonly known as plaque building up in the heart or blood vessel [1]. Worldwide statistics released by The AHA shows 2,200 deaths occur every day with 1 death for every 40 second was caused by CVD in 2017 [2]. On top of that, The World Health Organization (WHO) reported that about 98.9 deaths per 100,000 of the population in Malaysia was caused by CVD in 2012 [3]. The National Cardiovascular Disease announced that Malaysian citizen shows a younger age range of patient which is in average of age 55.9 to 59.1 years old detected with CVD compared to other inter-develop country [3]. This is because The National Cardiovascular Disease classified men of age less than 45 years old and women of age less than 55 years old as a young patient based on study done on recorded cardiac problem case between year 2007 to 2009 [3]. A report released by the AHA in early 2018 shows several contributing factor of CVD which includes smoking with 7.2 millions death in 2015, physical inactivity especially among children which shows shocking increment of $24.9 \%$ to $41.7 \%$ from 2009 to 2015 , and unhealthy lifestyle such as poor dietary habit which lead to obesity where meta-analysis conducted in 2016 shows higher risk of CVD for obese individual [4]. 
There are several type of CVD such as angina, heart attack and ischemic stroke. Each type of CVD tested with different procedure to diagnose the disease. This study narrow the scope by discussing procedure to diagnose ischemic stroke only. Normally, cardiologist would suggest patient with fair potential of ischemic stroke to undergo cardiac stress test to examine patient's heart condition [5]. Cardiac stress test is a test done by exerting stress to the patient's heart in form of exercise testing in order to observe any sign or symptom and evaluate the patient functional capacity as well as disease's severity and predict the effect of future medical and surgical therapy to be given to the patient [6]. The level of stress given according to the patient's capability to handle. Cardiac stress test is an option to determine prognosis or risk for patient with a history of heart problems. An accurate medical and surgical treatment can be prescribed if the physician has enough information on one's heart condition [6].

The result of cardiac stress test is measure in terms of maximal oxygen uptake $\left(V O_{2 \max }\right)$. There are three main method of measurement namely direct testing, indirect testing and self-testing [7]. Indirect testing and self-testing have several branches which differ by their procedure. There are Bruce protocol, Modified Bruce protocol and Naughton protocol for indirect testing $[8,9]$. Meanwhile, The Rockport Fitness Walking Test, Cooper Test and Heart Rate Ratio Method are several existing procedures for self-testing method [7]. These method of measurement differ by set of equipment used and procedures to obtain the final result. However, this paper will focus on The Rockport Fitness Walking Test (TRFWT) because it is the most suitable method for self-testing [10]. The $V_{2} \max$ value obtained utilized to predict the heart condition by converting the value into metabolic equivalent (MET) value. A metabolic equivalent or MET is defined as resting metabolic rate where amount of oxygen consumed at rest, sitting quietly on a chair is approximately $3.5 \mathrm{ml} \mathrm{O}_{2} / \mathrm{kg} / \mathrm{min}$ (1.2 $\mathrm{kcal} / \mathrm{min}$ for a $70 \mathrm{~kg}$ person) [11, 12]. Both TRFWT and MET will be discussed extensively in the following section.

Cardiac stress test has been recognized as an effective method in detecting abnormalities as well as determining heart functional capacity. However, there is no pre-diagnosis method available before conducting cardiac stress test procedure. Generally, abnormal heart activity appears unpredicted and cardiac stress test is tedious if it is done repeatedly. Moreover, there are a lot of patients lined up to undergo the procedure especially in government hospital due to limited access to the facilities. The problem can be tackled if there is a pre-diagnosis method available which assimilates the concept of pre-diagnosis device available today such as blood pressure test and blood sugar test. These devices enable users to do self-testing and the user will consult a doctor if the result is unsatisfying.

Therefore, a device was developed based on the concept of cardiac self-stress test and the result was converted into MET value to predict the heart condition. The idea was developed further by creating a device combining the use of application software which was built using MIT App Inventor 2 and hardware which consists of Arduino 101 and Sparkfun Heart Monitor AD8232. The result of the test was saved in a SD card for future reference. The device was named "i-STress device" and it aims to provide a pre-diagnosis result which is useful in deciding case priority.

\section{RESEARCH METHOD}

The implementation of the concept of cardiac self-stress test initiates the possibility of having portable cardiac stress test. This is because the test was usually conducted in a confined room using a set of equipment monitored by professionals. The suggested portable cardiac self-stress test device enable users to do selftesting with simple procedure, convenient and most importantly will yield a reliable pre-diagnosis result.

\subsection{Process Flow Chart}

Figure 1 shows the process flow chart of the device development. The main idea of the device is cardiac stress test which focus on self-testing procedure. The Rockport Fitness Walking Test procedure was chosen and the result was calculated by using Dolgener equation. The result interpreted in form of maximal oxygen uptake $\left(\mathrm{VO}_{2 \max }\right)$ and converted into metabolic equivalent (MET) value. Finally, the heart condition predicted by referring the final MET value to heart condition classification table.

\subsection{The Rockport Fitness Walking Test (TRFWT)}

Basically, self-testing method allow the patient to conduct the simple test by themselves and collect details which is then interpreted using specific equation provided by the procedure. The procedure of TRFWT only require the subject to maintain walking as fast as possible but not running for one mile or 1.6 kilometers distance $[13,14]$. The subject need to collect personal information such as weight, age, gender, duration to complete the test and record of the final heart rate upon the test completion. These information substituted into parameters in the equation to find $V O_{2 \max }$. This procedure chosen over the other existing procedure because it is simple to execute compared to Cooper Test and Heart Rate Ratio Method. 
The Cooper Test only require the subject to run but it is impossible for a patient suspected with a heart problem to run continuously for 12 minutes [10]. Even though the Cooper Test give a convincing outcome, the application is more practical for cardiorespiratory fitness monitoring rather than medical purpose due to the procedure [15]. On the other hand, the Heart Rate Ratio Method consume a lot of time which is around 48 hours to complete because the subject need to follow a set of procedure on a treadmill and record $H R_{\max }$ and $H R_{\text {rest }}$ to determine the final result [10]. Besides, the test can be conducted outdoor and the test time is flexible as long as the duration of test completion recorded.

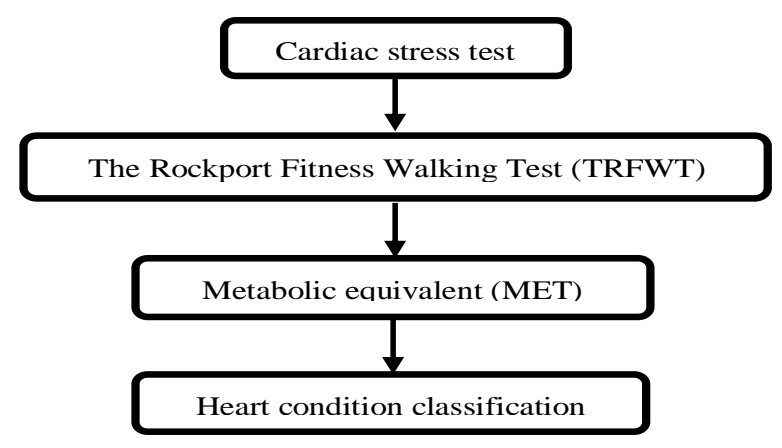

Figure 1. The process flow chart

\subsubsection{The Dolgener equation}

There are two main equations that are mainly used in calculating the final value of $V O_{2 \max }$ when using TRFWT procedure. These equations are namely Kline equation [16, 17] and Dolgener equation [17]. Originally, the Kline equation was used to calculate the result of test. However, the Dolgener equation is used to calculate the final result because the body weight measurement unit is in kilogram which is International System of Unit (SI unit) instead of Kline equation which use pound to measure the body weight. The subject is required to walk briskly instead of walking as fast as possible which is hard to maintain $[19,20]$. Besides, the distance needed to be complete only a quarter mile or 400 meters instead of one mile because studies have shown that the heart reach steady state after first three minute of walking in steady pace [18-20]. The Dolgener equation is shown as follows [18]. The final result only providing estimation value of $\mathrm{VO}_{2 \max }$ and the value can be refer to standard table as shown in Table 1 to determine cardiovascular fitness.

$\mathrm{VO}_{2 \max }(\mathrm{ml} / \mathrm{kg} / \mathrm{min})=94.6440-(0.0819 * \mathrm{BM})-(0.3232 * \mathrm{~A})+(8.4073 * \mathrm{G})-(1.6157 * \mathrm{~T})-(0.1146 * \mathrm{HR})$

Where;

$\mathrm{BM}=$ Body mass (kilograms)

$\mathrm{A}=$ Age

$\mathrm{G} \quad=$ Gender $($ Female $=0$, Male $=1)$

$\mathrm{T} \quad=$ Time complete (minute)

$\mathrm{HR}=$ Heart rate

Table 1. Men and Women Cardiovascular Fitness Rating [21]

\begin{tabular}{cccccc}
\hline CLASS & \multicolumn{5}{c}{ MEN } \\
\hline V.POOR & $\leq 38-19$ & $30-39$ & $40-49$ & $50-59$ & $60-69$ \\
POOR & $38.1-42.1$ & $36.7-40.9$ & $34.6-38.3$ & $31.1-35.1$ & $27.4-31.3$ \\
FAIR & $42.2-45.6$ & $41.0-44.3$ & $38.4-42.3$ & $35.2-38.2$ & $31.4-34.9$ \\
GOOD & $45.7-51.0$ & $44.4-48.8$ & $42.4-46.7$ & $38.3-43.3$ & $35.0-39.4$ \\
GREAT & $51.1-56.1$ & $48.9-54.2$ & $46.8-52.8$ & $43.3-49.6$ & $39.5-46.0$ \\
SUPERB & $\geq 56.1$ & $\geq 54.2$ & $\geq 52.8$ & $\geq 49.6$ & $\geq 46.0$ \\
\hline AGE & & \multicolumn{3}{c}{ WOMEN } & $50-69$ \\
CLASS & $18-29$ & $30-39$ & $40-49$ & $50-59$ & $\leq 23.7$ \\
V.POOR & $\leq 31.6$ & $\leq 29.9$ & $\leq 28.0$ & $\leq 25.5$ & $23.7-26.5$ \\
POOR & $31.6-35.4$ & $29.9-33.7$ & $28.0-31.5$ & $25.5-28.6$ & $26.6-29.0$ \\
FAIR & $35.5-39.4$ & $33.8-36.7$ & $31.6-35.0$ & $28.7-31.3$ & 29.132 .2 \\
GOOD & $39.5-43.9$ & $36.8-40.9$ & $35.1-38.8$ & $31.4-35.1$ & $32.3-36.8$ \\
GREAT & $44.0-50.1$ & $41.0-46.8$ & $38.9-45.1$ & $35.2-39.8$ & $\geq 36.8$ \\
SUPERB & $\geq 50.1$ & $\geq 46.8$ & $\geq 45.1$ & $\geq 39.8$ & \\
\hline
\end{tabular}

Portable cardiac self-stress test device development based on metabolic... (Whomaira binti Abdul Samad) 


\subsection{Metabolic Equivalent (MET)}

The value of $V O_{2 \max }$ obtained can be use to predict the heart condition by converting $V O_{2 \max }$ value into metabolic equivalent (MET) value [11]. The MET formula is shows as follows:

$$
\text { Functional capacity }(\mathrm{MET})=\frac{\text { Number of oxygen uptake }\left(\mathrm{ml} \mathrm{O}_{2}\right)}{3.5 \mathrm{ml} \mathrm{O}_{2}}
$$

The MET value is commonly use in quatifying the value of physical activites. Based on the guidelines, light, intermediate and heavy activities gives value <3.0 MET, 3.0-5.9 MET, and $\geq 6.0$ MET respectively [22]. Apart from its usual application, MET value use for classification and predict the condition of heart. The classification and prediction table is called New York Heart Association (NYHA) Classification $[9,23]$. The classification is divided into four classes and Table 2 shows the heart condition for each classes. The classification have been tested multiple times since it was introduced and the prediction gives high validity in term of results $[24,25]$.

\begin{tabular}{ccc}
\multicolumn{2}{c}{ Table 2. New York Heart Association (NYHA) Classification [9] } \\
\hline Mets Value (METS) & Classification & Condition \\
\hline More than 7 & Class I & No impairment \\
5 to 7 & Class II & Minimal functional impairment \\
3 to 4 & Class III & Moderate impairment \\
Less than 3 & Class IV & Severe impairment \\
\hline
\end{tabular}

\section{RESULTS AND ANALYSIS}

Based on the concepts explained previously, a design of the overall functioning system was created. The device divided into two part which is the hardware and application software development. It is mentioned earlier that the application software built by using MIT Apps Inventor 2 and the hardware built based on Arduino 101 and Sparkfun Heart Monitor AD8232. The hardware was named Black Box and the software named "i-STress Apps". The apps provide two test mode for user; Black Box and Palpation. These modes will be explained extensively in the following section.

\subsection{I-STress Apps}

The i-STress Apps was developed by using MIT Apps Inventor 2. The apps prompt the user to complete the test according to TRFWT procedure. When the user activated the apps, the first screen will appear as shown in Figure 2.

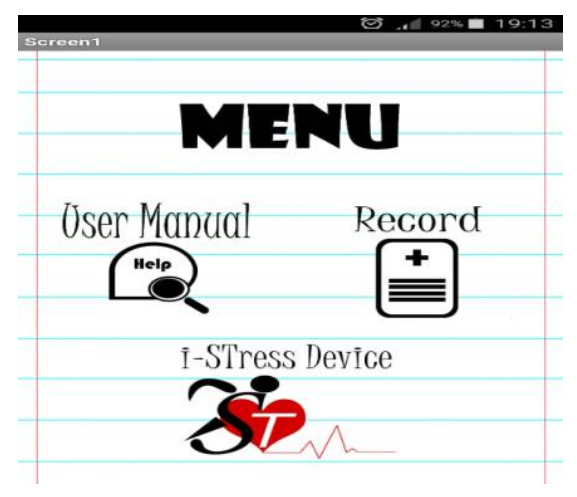

Figure 2. The first screen

Figure 2 is the main menu which consists of "User Manual", "Record" and "i-STress Device". First and foremost, the "User Manual" shows step by step procedure of completing the test. The user manual then provides a guide to use item "i-STress Device" and "Record". When the "i-STress Device" selected, the user instructed to choose either one of the test mode as shown in Figure 3. 


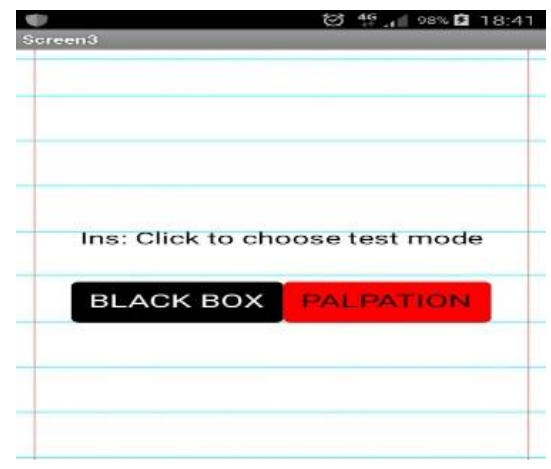

Figure 3. The second screen (test mode)

\subsubsection{Black Box}

The "Black Box" mode require user to connect to an extension device to complete the test. The extension device called "Black Box" was consist of Sparkfun single lead heart monitor AD8232, Arduino 101 and SD card module. The hardware connection sketched by using software Fritzing shown in Figure 4.

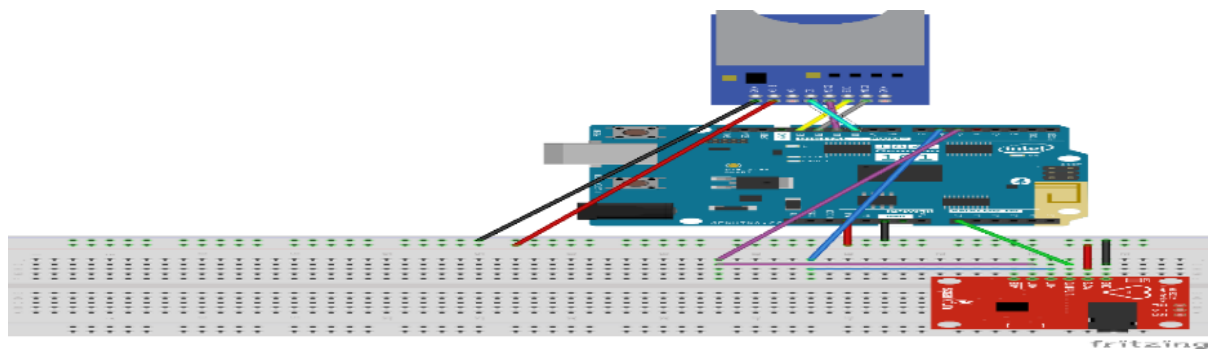

Figure 4. Hardware connection

Initially, the user have to attach electrode on their limbs and connect it to Sparkfun single lead heart monitor's input jack. It is highly recommended that the user rub the alcohol swab on the skin before attaching the electrode to remove the dead skin. The user was fairly reminded to turn on the Bluetooth and Global Positioning System (GPS) on their device so that it can be connected to the Black Box. Figure 5 shows the input-process-output (I-P-O) layout of the overall system.

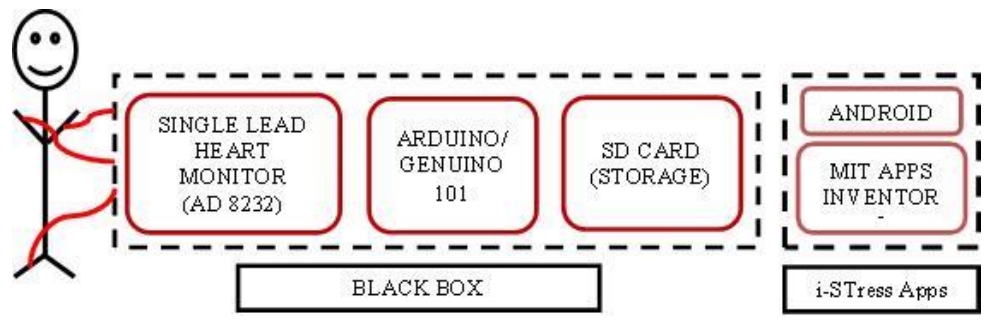

Figure 5. The Input-Process-Output (I-P-O) layout

The "Black Box" mode require the user to do the Black Box set up and skin preparation before selecting the mode in the apps. Once the user ready, the following Figure 6 will appear. There are two item shown in this screen; the heart rate reading and timer. 


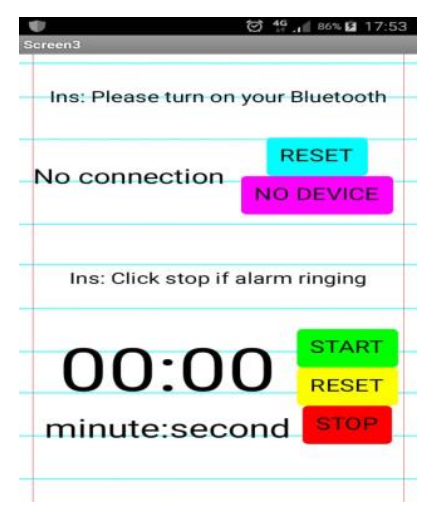

Figure 6. Third screen (first stage)

The user reminded to turn on their Bluetooth and GPS function. User needs to click on button "RESET" as shown in Figure 6 to find the Black Box. The purple button will change from "NO DEVICE" to "Choose a device" when hardware was detected and user needs to click on the purple button to connect to hardware. Once connected, the value of heart rate which is in beat per minute (BPM) will appear. The user may start the test by clicking on the "START" button and the timer will be activated. The timer will record the duration of test in minute and second. There are clear instructions to the user to stop the timer if the alarm is ringing. The alarm was actually triggered by total distance traveled by the user. It is set to ring when the user travel for 400 meter or a quarter mile which is the distance traveled required to use The Dolgener equation. If the user needs to restart the test, a "RESET" button is available. The test only require the user to walk briskly until the alarm ringing. The "NEXT" button visible if the button "STOP" clicked and user will be able to proceed to the next stage of the test.

The user advanced to second stage by providing the needed information as shown in Figure 7. The information required are weight, age, gender, heart rate and time taken to complete the test. These informations needed to produce the result based on the Dolgener equation as shown previously. The user will get the final result by clicking the button "CALCULATE" and the test come to the final stage which is displaying result as shown in Figure 8.

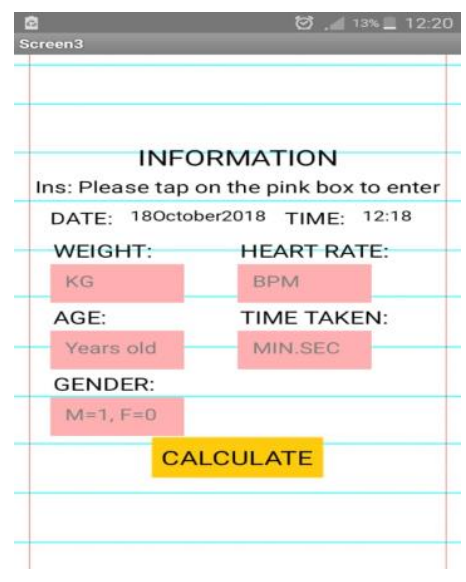

Figure 7. Third screen (second stage)

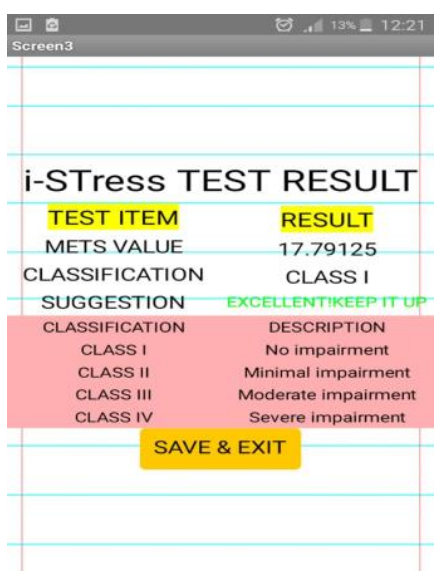

Figure 8. Third screen (Third stage)

The result item includes METS value, heart condition classification, and suggestion for future action. The suggestion section differed by color for each classes to show the level of urgency to consult a doctor for further action. There are four classes of heart condition namely Class I, Class II, Class III and Class IV and a simple description on the heart condition is attached. Each class will display different suggestion text for user's further action. The text message displayed with corresponding class is shown in Table 3. The result will be saved in "Record" and each record includes the time and date of the test, MET value, heart condition classification, abnormality, and suggestion for further action. 
Table 3. Classification and Message Displayed

\begin{tabular}{cr}
\hline Classification & Message \\
\hline Class I & Excellent! Keep it up! \\
Class II & Precaution! Practice healthy lifestyle! \\
Class III & Please consult a doctor! \\
Class IV & Extremely advice to consult a doctor! \\
\hline
\end{tabular}

User could review quickly on the heart condition by referring to the table of heart classification with corresponding description of each classes. The test was successful and user may save the result and exit to the main menu by clicking to the "SAVE \& EXIT" Button. Third screen as shown in Figure 9.

As the test progressing, the electrocardiogram (ECG) signal was recorded by the Black Box and saved in SD card. These activities were controlled by Arduino 101 and once the test completed, the result of the test was saved in the SD card mounted on the phone. Overall, there are two data saved on two different places; the ECG signal saved on the hardware SD card and the cardiac self-stress test result on the phone SD card. The result of the previous test can be review under the item "RECORD" as shown in the main menu. The sample of recorded result shown Figure 10. The record include the date and time of the test as well as the final result of the test.

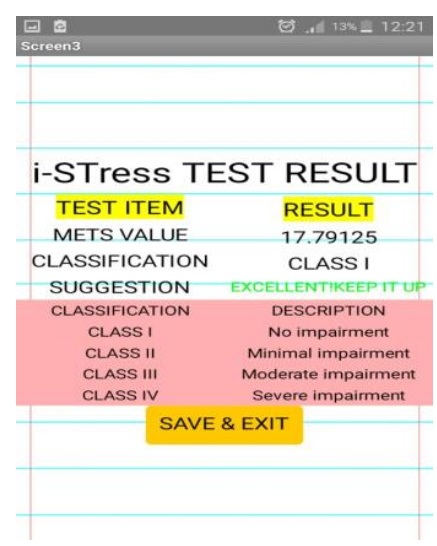

Figure 9. Third screen (Third stage)

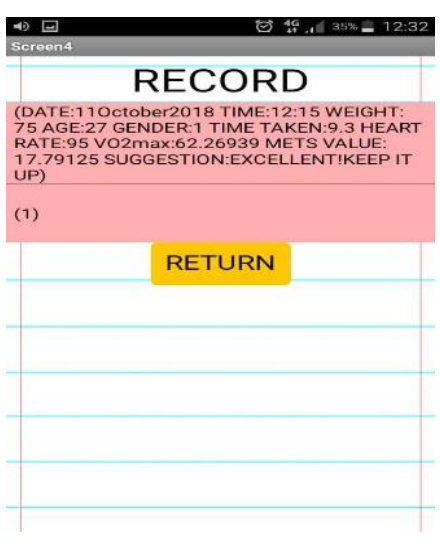

Figure 10. Previous result record

\subsubsection{Palpation}

Palpation mode differ on the first stage of the test. The palpation mode does not require the user to own the Black Box. Figure 11 shows the user may directly start the test by clicking the button "START" to activate the timer and stop if the alarm ringing. It is fairly reminded that the user need to make sure that the GPS function is activated beforehand. User only required to walk briskly during the test and it is highly advisable for user to warm-up and dress comfortably to do the test.

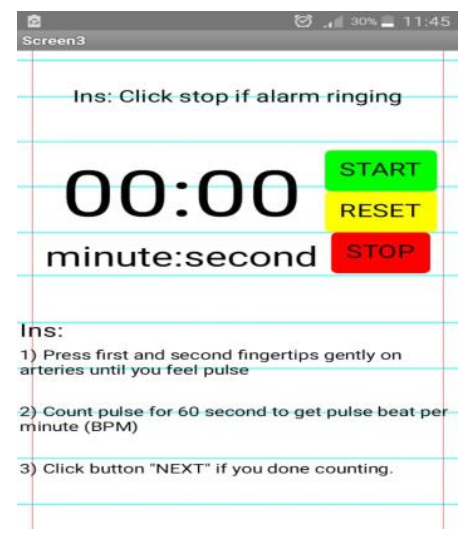

Figure 11. Third screen (first stage palpation mode) 
The user need to follow three simple instruction after clicking the button "STOP" to get the heart rate reading. The user instructed to place index and middle fingertips gently on the radial arteries which is place just below the thumb and count the pulse for 60 second to get the pulse beat per minute (BPM). Then, proceed to the next stage by clicking the button "NEXT". Second and third stage of the test similar to the stages explained previously. The test result can be review in the item "RECORD" on the main menu and saved in SD card for future reference.

\section{CONCLUSION}

In conclusion, the concept of portable cardiac self-stress testing can be developed by implementing the self-stress test procedure which in this case by using The Rockport Fitness Walking Test procedure and the result is even extended to predict the heart condition by using metabolic equivalent (MET) value. When reliable result obtained, a pre-diagnosis reference created and it is useful in deciding case priority for real stress test which eventually will shorten the queue for higher risk patient to use the facilities in the government hospital. It is fairly reminded that the concept of the idea is self-screening oriented and the development of i-STress system as a portable cardiac self-stress test may be a stepping stone to enhance the available diagnosis method.

\section{ACKNOWLEDGEMENTS}

This research is supported by Universiti Teknologi Malaysia Research and Development Fund (UTMF), with Research Grant No. R.J130000.7323.4J208.

\section{REFERENCES}

[1] The American Heart Association, "What is Cardiovascular Disease?," The American Heart Association, United States, 2017.

[2] Benjamin EJ, Blaha MJ, Chiuve SE, "Heart Disease and Stroke Statistic 2017 At-a-Glance," American Heart Society, United States, 2017.

[3] Ang Choon Seong, Chan Kok Meng John, "A Review of Coronary Artery Disease Research in Malaysia," Med. J Malaysia, pp. 42-57, 2016.

[4] Emilia J. Benjamin, Salim S. Virani, Clifton W. Callaway \& et. al, "Heart Disease and Stroke Statistics - 2018 Update (A Report from The American Heart Association)," The American Heart Association, Texas, 2018.

[5] Kathryn K. Garner, William Pomeroy, James J. Arnold, "Exercise Stress Testing: Indications and Common Questions," American Family Physician, vol. 96, no. 5, pp. 293-300, 2017.

[6] George D. Harris, Russell D. White, "Performance of The Exercise Test," in Exercise Testing for Primary Care and Sports Medicine, Kansas City, Springer Science+Business Media, 2009, pp. 23-42.Chris Dinesen Rogers, "Equipment Used to Measure Oxygen Uptake During Exercise," 7 Febuary 2014. [Online]. Available: www.livestrong.com/article/424672-equipment-used-to-measure-oxygen-uptake-during-exercise/.

[7] Chris Dinesen Rogers, "Equipment Used to Measure Oxygen Uptake During Exercise," 7 Febuary 2014. [Online]. Available: www.livestrong.com/article/424672-equipment-used-to-measure-oxygen-uptake-during-exercise/.

[8] Robert A. Robergs, "Simplified Method and Program for Incremental Exercise Protocol Development," Journal of Exercise Physiology, pp. 1-23, 2007.

[9] John N. Hamaty, "Cardiac Stress Testing," South Jersey, 2008.

[10] Whomaira Abdul Samad, Mohd Afzan Othman, Norlaili Mat Safri, "Comparison on Measurement Method of Maximal Oxygen Uptake during Cardiac Stress Test," Biomedical Electronics and Informatics, vol. 9, no. 3-9, pp. 113-117, 2017.

[11] M. Jette, K. Sidney, G. Blumchen, "Metabolic Equivalents (METS) in Exercise Testing, Exercise Prescription, and Evaluation of Functional Capacity," Clin. Cardiol, pp. 555-565, 1990.

[12] Andrew P.Hills, Najat Mokhtar, Nuala M. Byrne, "Assessment of Physical Activity and Energy Expenditure: An Overview of Objective Measure," Frontier in Nutrition, vol. 1, no. 5, pp. 1-16, 2014.Rockport Shoes Walking Institute, "Rockport One Mile Walking Test," Rockport Walking Institute, Malboro, 1986.

[13] Rockport Shoes Walking Institute, "Rockport One Mile Walking Test," Rockport Walking Institute, Malboro, 1986.

[14] Brian Mackenzie, "Rockport Fitness Walking Test," in 101 Performance Evaluation Test, London, Electric Word plc, 2005, pp. 33-34.

[15] Daniel Mayorga-Vega, Raul Bocanegra-Parilla, Martha Ornelas, Jesus Viciana, "Criterion-Related Validity of The Distance- and Time-Based Walk/Run Field Test for Estimating Cardiorespiratory Fitness: A Sytematic Review and Meta-Analysis," PLOS ONE, vol. 11, no. 3, p. p. e 0151671, 2016.

[16] Greg M. Kline, John P. Porcari, Robert Hintermeister, Patty S. Freedson, Ann Ward, Robert F. Mccarron, Jessica Ross, James M. Rippe, "Estimation of VO2max From One-Mile Track Walk, Gender, Age, and Body Weight," Medicine and Science in Sports and Exercise, pp. 253-259, 1987. 
[17] Christopher Paul Keller, "Validation of The 1-Mile Walking Test in Young Adults at Maximal and Submaximal Walking Intensities," Montana State University, Montana, 2002.

[18] Dolgener F.A., Hensley L.D., Marsh, J.J., Fjelstul, J.K., "Validation of The Rockport Filtness Walking Test in College Males and Females," Research Quarterly for Exercise and Sports, pp. 152-158, 1994.

[19] James D. George, Gilbert W. Fellingham, A. Garth Fisher, "A Modified Version of The Rockport Fitness Walking Test for College Men and Women," Reasearch Quarterly for Exercise for Exercise and Sport, pp. 205-209, 1998.

[20] Freddie D. McConnell II, "Modifications of The Rockport Fitness Walking Test," Bell \& Howell Information and Learning Company, Tennessee, 2001. Thomas D. Fahey, "Cardiorespiratory Endurance," in Fit and Well: Core Concepts and Labs in Physical Fitness and Wellness, McGraw-Hill Ryerson, 2007, pp. 57-90.

[21] Thomas D. Fahey, "Cardiorespiratory Endurance," in Fit and Well: Core Concepts and Labs in Physical Fitness and Wellness, McGraw-Hill Ryerson, 2007, pp. 57-90. Marcio de Almelda Mendes, Inacio da Sliva, Vargilio Ramires, Felipe Reichert, Rafaela Martins, Rodrigo Ferreira, Elaine Tomasi, "Metabolic Equivalent of Task (METs) Thresholds As An Indicator of Physical Activity Intensity," PLOS ONE, p. p. e0200701, 2018.

[22] Marcio de Almelda Mendes, Inacio da Sliva, Vargilio Ramires, Felipe Reichert, Rafaela Martins, Rodrigo Ferreira, Elaine Tomasi, "Metabolic Equivalent of Task (METs) Thresholds As An Indicator of Physical Activity Intensity," PLOS ONE, p. p. e0200701, 2018.

[23] Chronic Heart Failure Guidelines Expert Writing Panel, "New York Heart Association (NYHA) Classification," National Heart Foundation of Australia and The Cardiac Society of Australia and New Zealand, Australia, 2011.

[24] Ponrathi Athilingam, Rita D'aoust, Cheryl Zambroski, Susan C. McMillan, Frances Sahe bze mani, "Predictive Validity of NYHA and ACC/AHA Classifications of Physical and Cognitive Funtioning in Heart Failure," International Journal of Nursing Science, vol. 3, no. 1, pp. 22-32, 2013.

[25] Charlene Bredy, Margherita Ministeri, Alexander Kempny, Rafael Alonso-Gonzalez, Lorna Swan, Anselm Uebing, Gerhard-Paul Diller, Michael A.Gatzoulis, Konstantinos Dimonopoulos, "New York Heart Association (NYHA) Classification In Adults With Congenital Heart Disease: Relation To Objective Measures of Exercise And Outcome," European Heart Journal, vol. 4, no. 1, pp. 51-58, 2018.

\section{BIOGRAPHIES OF AUTHORS}

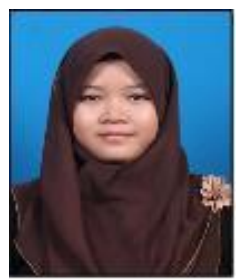

Whomaira Binti Abdul Samad received her degree in Bachelor of Engineering (ElectricalElectronics) with honours from Universiti Teknologi Malaysia and currently pursuing Master of Philosophy (Electrical Engineering). Her main interest of study is medical electronics specifically in electrocardiogram.

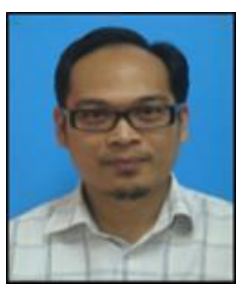

Mohd Afzan Bin Othman is a senior lecturer in Department of Electronics and Computer Engineering, School of Electrical Engineering, Faculty of Engineering, Universiti Teknologi Malaysia. He is also a researcher in BioMedical Instrumentation and Electronics (bMIE) of Infocomm Research Alliance. His field of interest including Electrocardiogram (ECG), cardiac monitoring and image and signal processing.

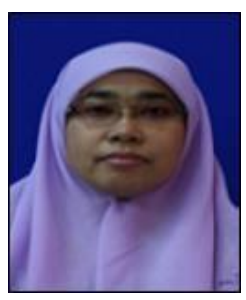

Norlaili Binti Mat Safri received her Doctor of Philosophy in Systems and Information from Kumamoto University, Japan. She is currently holding post as Associate Professor at Department of Electronic and Computer Engineering in School of Electrical Engineering, Faculty of Engineering, Universiti Teknologi Malaysia. Her interest focus on medical electronics and have huge involvement in designing Biosystem Modelling, Biomedical Signal Processing and Clinical Engineering courses at School of Electrical Engineering.

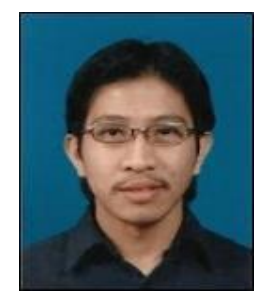

Mohd Azhar Bin Abdul Razak was a PhD holder in Biomedical Microengineering, School of Engineering, University of Surey, United Kingdom. He is currently a senior lecturer lecturer in Department of Electronics and Computer Engineering, School of Electrical Engineering, Faculty of Engineering, Universiti Teknologi Malaysia and Head of Group in Biomedical Instrumentation and Electronics (bMIE). 